Edited by Kiriakos Xenitidis and
Colin Campbell
Contents
- Behavioural and psychiatric symptoms in people
with dementia admitted to acute hospitals
- More data on speed of remission with ECT in
geriatric depression
- The 'unknown' safety concern for aripiprazole
once monthly

\section{Behavioural and psychiatric symptoms in people with dementia admitted to acute hospitals}

We would like to commend Sampson et al ${ }^{1}$ on undertaking the difficult task of identifying and monitoring behavioural and psychological symptoms of dementia (BPSD) in people admitted to acute medical wards. The authors have also done their best to untangle the BPSD syndrome from similar clinical symptomatology seen in delirium, which still remains an ongoing conundrum for many of us working in liaison psychiatry. The study not only provides a wealth of information, but also raises a number of issues about how BPSD presentation might differ in older people when admitted to acute medical settings and how it influences their outcomes.

The authors used the Behavioral Pathology in Alzheimer's Disease scale (BEHAVE-AD), ${ }^{2}$ which has been widely used to detect behavioural problems in people with dementia, in particular Alzheimer's disease. However, this scale has a number of shortcomings, neglecting some important symptoms of dementia, such as apathy, irritability and/or disinhibition, ${ }^{3}$ that are frequently present in dementia. The low-medium BPSD scores (2.6-4.4 mean BEHAVE-AD severity) are somewhat surprising, since the majority of the enrolled participants had higher Functional Assessment Staging Test (FAST) ${ }^{4}$ staging, corresponding to more advanced stages of dementia. Such low BPSD scores are usually associated with mild cognitive impairment. ${ }^{2}$ Only five individuals coming from an 'other' place of residence (continuing NHS care?) had substantially higher BPSD severity scores. Similarly, the severity of the dementia (as measured via the FAST) did not influence BPSD scores, suggesting that either the medical problems modified the BPSD presentation, or the BPSD were pharmacologically managed. The only factor to have a significant effect on BPSD was presence of delirium, thus highlighting the difficulties in routine clinical settings of differentiating between BPSD and clinical symptoms of delirium.

Nevertheless, Sampson et al's work undoubtedly indicates that hospitals make people with dementia worse, trebling their paranoia and delusional beliefs, causing hallucinations, making them more aggressive and disturbed, and substantially worsening their moods and anxieties. These findings support the public's widespread beliefs that hospitals are dangerous places, not only filled with sick people and germs, but with a wide potential for something to go amiss in lieu of wrong. And this 'wrong' ranges from having newly acquired diagnosis of dementia when physically unwell to worsening BPSD, ${ }^{1}$ further complicating their polypharmacy and making them more frail and with poorer functional outcomes, ${ }^{5}$ as well as increasing their likelihood of death. ${ }^{1}$ Not surprisingly, this also affects their formal and informal caregivers. There is a striking discord between the severity of the recorded BPSD and the caregiver's distress, arguing that the problems around the escalating in-hospital behavioural changes are much more serious than the physical illness itself.

Many of the highlighted BPSD could be easily regulated with non-pharmacological approaches, including better orientation, information and knowing our patients. What is happening to make our hospitals more dementia-friendly? A number of hospitals have already introduced dementia-friendly wards that should be fully equipped with the professional experts in dementia care. However, surprisingly, there is a void of research evidence regarding how the newly introduced dementia-friendly policy in acute medical settings influences the behaviour of people with dementia. Since most of the people with BPSD come from residential and nursing care, ${ }^{1}$ one wonders whether there should be another way of introducing dementia-friendly management. Perhaps an 'in-home health dementia care' approach would avoid (unnecessary) hospital admissions, and involve medical care professionals treating people with dementia in their own home whenever possible. This would reduce substantially not only the distress that both people with dementia and their caregivers face when in the acute medical setting, but also reduce hospital admissions, and thus result in substantial financial savings. The data from a recent study ${ }^{6}$ on people with advanced dementia stages prove that this can be successfully done, stressing that managing the distress, rather than behaviours that challenge, is central when treating people with dementia. We now need to take these lessons on board and implement them not only within our acute medical wards, but also adapt them to use in the community.

1 Sampson EL, White N, Leurent B, Scott S, Lord K, Round J, et al. Behavioural and psychiatric symptoms in people with dementia admitted to the acute hospital: prospective cohort study. Br J Psychiatry 2014; 205: 189-96.

2 Reisberg B, Auer SR, Monteiro IM. Behavioral pathology in Alzheimer's disease (BEHAVE-AD) rating scale. Int Psychogeriatr 1996; 8 (suppl 3): 301-8.

3 Robert P, Ferris S, Gauthier S, Ihl R, Winblad B, Tennigkeit F. Review of Alzheimer's disease scales: is there a need for a new multi-domain scale for therapy evaluation in medical practice? Alzheimers Res Ther 2010; 2: 24.

4 Reisberg B. Functional assessment staging (FAST). Psychopharmacol Bull 1988; 24: 653-9.

5 Runganga M, Peel NM, Hubbard RE. Multiple medication use in older patients in post-acute transitional care: a prospective cohort study. Clin Intervent Aging 2014; 9: 1453-62.

6 Treloar A, Crugel M, Adamis D. Palliative and end of life care of dementia at home is feasible and rewarding. Results from the 'Hope for Home' study. Dementia 2009; 8: 335-47.

Elizabeta B. Mukaetova-Ladinska, Senior Lecturer in Old Age Psychiatry, Institute of Neuroscience, Newcastle University, Newcastle University Institute for Ageing and Newcastle Liaison Team for Older Adults, Tyne and Wear NHS Foundation Trust, Newcastle upon Tyne. Email: Elizabeta.Mukaetova-Ladinska@ncl.ac.uk. Ann Scully, Consultant in Old Age Psychiatry, Newcastle Liaison Team for Older Adults, Tyne and Consultant in Old Age Psychiatry, Newcastle Liaison
Wear NHS Foundation Trust, Newcastle upon Tyne.

doi: 10.1192/bjp.206.2.166

Author's reply: Mukaetova-Ladinska \& Scully's comments on the challenges of conducting clinical research involving people with dementia on acute hospital wards ${ }^{1}$ raise the issue of whether BPSD may present differently in the acute hospital.

In our study, those with more severe dementia were unwell, often bed-bound, and may have been less able to display BPSD (e.g. wandering or pacing). Additionally, $12 \%$ of our cohort was taking an antipsychotic (details available from the authors on request), and many of these patients had more severe dementia. We note the problem of recall bias in staff who may report problems that are harder to manage; for example, depression in 
those with severe dementia may not have been recognised. Although BPSD were not associated with severity of dementia assessed by the FAST, ${ }^{2}$ we notice a possible difference between those of stages 3-5 (mean total BEHAVE-AD score over admission: 2.4) and the more severe stages (means 3.6, 3.4 and 3.7, respectively, for stages $6 \mathrm{a}-\mathrm{c}, 6 \mathrm{~d}-\mathrm{e}$ and $7 \mathrm{a}-\mathrm{f})$.

We agree the BEHAVE-AD scale has shortcomings; for example, it misses apathy and disinhibition. ${ }^{3}$ Our choice was pragmatic, based on ease of administration and available staff time. The Neuropsychiatric Inventory has more detailed items on agitation and aggression, but we also used the CohenMansfield Agitation Inventory to characterise agitated behaviour (details available from the authors on request) and wished to avoid duplicating data collection. We would like to highlight that most of our cohort did not come from residential or nursing care; $67 \%$ were admitted from their own home (Table 2). ${ }^{1}$

Although admission is overall a negative experience, the precipitating illness may require hospital treatment. We had no data on BPSD prior to admission or how they would have evolved in another setting. Teasing out which elements of the admission have the strongest influence on poor outcomes, or whether the physical illness causing the admission produces negative effects, would require further investigation. Unfortunately, the answers to these questions will be complex and methodologically challenging to define properly.

There is recent evidence that improving the hospital environment for people with dementia is worthwhile. ${ }^{4}$ We hope our paper provides information to inform more effective interventions.

1 Sampson EL, White N, Leurent B, Scott S, Lord K, Round J, et al. Behavioura and psychiatric symptoms in people with dementia admitted to the acute hospital: prospective cohort study. Br J Psychiatry 2014; 205: 189-96.

2 Reisberg B. Functional assessment staging (FAST). Psychopharmacol Bull 1988; 24: 653-9.

3 Reisberg B, Borenstein J, Salob SP, Ferris SH, Franssen E, Georgotas A Behavioral symptoms in Alzheimer's disease: phenomenology and treatment. J Clin Psychiatry 1987; 48 (suppl): 9-15.

4 Harwood RH, Goldberg SE, Whittamore KH, Russell C, Gladman JR, Jones RG, et al. Evaluation of a Medical and Mental Health Unit compared with standard care for older people whose emergency admission to an acute general hospital is complicated by concurrent 'confusion': a controlled clinical trial. Acronym: TEAM: Trial of an Elderly Acute care Medical and mental health unit. Trials 2011; 12: 123.

Elizabeth L. Sampson, Marie Curie Palliative Care Research Department, Division of Psychiatry, University College Medical School and Barnet, Enfield and Haringey Menta Health Trust Liaison Psychiatry Team, North Middlesex University Hospital, London. Email: e.sampson@ucl.ac.uk

doi: $10.1192 / \mathrm{bjp} .206 .2 .166 \mathrm{a}$

\section{More data on speed of remission with ECT in geriatric depression}

We appreciate the important contribution of Spaans et al ${ }^{1}$ to the evidence that electroconvulsive therapy (ECT) is a rapidly acting treatment in geriatric depression. Their data are a reminder that, despite the recent excitement about other neuromodulation modalities for the treatment of depression, ECT remains a standard and vital treatment for our most seriously ill patients, particularly those in the geriatric age group. We would like to add data about the speed of ECT remission in geriatric depression from the ongoing National Institute of Mental Health (NIMH)supported multicentre trial, Prolonging Remission in Depressed Elderly (PRIDE, ClinicalTrials.gov Identifier: NCT01028508).

Our group has just completed enrolment of 237 patients in phase 1 of a trial in which patients with unipolar depression over 60 years of age receive a course of ultra-brief pulse right unilateral ECT augmented with venlafaxine. (Phase 2 of the trial is random allocation to venlafaxine plus lithium or venlafaxine plus lithium plus flexible maintenance ECT. This phase of the trial will be completed in the next 3 months.) The cohort of 133 remitters in phase 1 required a mean of 7.3 (s.d. = 3.1) ECT sessions to reach remission, defined as a Hamilton Rating Scale for Depression (HRSD-24) score of $\leqslant 10$ on two consecutive occasions (personal communication, R. Knapp). Because ECT was administered three times a week in our study, seven treatments approximate 2.5 weeks until remission, a time comparable to that reported by Spaans et al.

In our previous study, comparing the efficacy of the three standard electrode placements in ECT, ${ }^{2}$ the mean number of ECT sessions needed to achieve remission in patients over 60 years of age was also consistently low: bi-temporal (5.5, s.d. $=2.2$, $n=19$ ), bi-frontal (5.4, s.d. $=2.1, n=11$ ), right unilateral brief pulse $(5.1$, s.d. $=2.1, n=19)$. Speed of response takes on added importance when patients are urgently ill and present with severe suicidal urges, agitation, psychosis, or malnutrition from profound depression. Because of its unsurpassed efficacy and now better-documented speed of response in geriatric depression, ECT should no longer be relegated to last place in treatment algorithms for severe depression. ${ }^{3}$ Finally, it should be noted that in both Spaans et al and the PRIDE study, newer techniques allow practitioners to prescribe ECT in a form that is more tolerable for patients than in the past. ${ }^{4}$

1 Spaans HP, Sienaert P, Bouckaert F, van den Berg JF, Verwijk E, Kho KH, et al. Speed of remission in elderly patients with depression: electroconvulsive therapy v. medication. Br J Psychiatry 2015; 206 67-71.

2 Kellner $\mathrm{CH}$, Knapp R, Husain MM, Rasmussen $\mathrm{K}$, Sampson $\mathrm{S}$, Cullum M, et al. Bifrontal, bitemporal and right unilateral electrode placement in ECT: randomised trial. Br J Psychiatry 2010; 196: 226-34.

3 Eranti SV, McLoughlin DM. Electroconvulsive therapy - state of the art. Br J Psychiatry 2003; 182: 8-9.

4 Prudic J. Strategies to minimize cognitive side effects with ECT: aspects of ECT technique. J ECT 2008; 24: 46-51.

Charles H. Kellner, Professor, Department of Psychiatry, Icahn School of Medicine USA. Email: charles.kellner@mssm.edu. Emma T. Geduldig, Clinical Research Coordinator, Department of Psychiatry, Icahn School of Medicine, Rebecca G. Knapp, Professor Emerita, Department of Public Health Sciences, Medical University of South Carolina, Robert C. Young, Professor, Department of Psychiatry, Weill Medical College of Cornell University, Richard D. Weiner, Professor, Department of Medical College of Cornell University, Richard D. Weiner, Professor, Department of
Psychiatry and Behavioral Sciences, Duke University School of Medicine, Robert M. Greenberg, Chief, Geriatric Psychiatry, Lutheran Medical Center, Joan Prudic, Clinical Professor of Psychiatry, New York State Psychiatric Institute and the College of Physicians and Surgeons of Columbia University, W. Vaughn McCall, Professor and Case Distinguished Chairman, Department of Psychiatry and Health Behavior, Georgia Regents University, Georgios Petrides, Associate Professor, Department of Psychiatry, Hofstra North Shore-LIJ School of Medicine at Hofstra University, Mustafa M. Husain, Professor, Department of Psychiatry and Behavioral Sciences, Duke University School of Medicine, Matthew V. Rudorfer, Program Chief, Somatic Treatments Program, National Institute of Mental Health, Sarah H. Lisanby, JP Gibbons Professor and Chair, Department of Psychiatry and Behavioral Sciences, Duke University School of Medicine, USA.

doi: 10.1192/bjp.206.2.167

Authors' reply: We agree that the superior efficacy and faster onset of action of ECT compared with other treatment modalities warrants the earlier application of ECT in the treatment of elderly patients suffering from severe depression. The growing evidence of superior efficacy in the subgroup of elderly patients ${ }^{1}$ suggests the existence of distinctive subgroups with individual, clinical, cognitive and genetic parameters predicting response or non-response, as well as the emergence of side-effects. An exploratory study on clinical and cognitive profiles that predict early and complete remission with a Clinical Global Impression of Severity of 1 within 2 weeks of treatment has been submitted for publication. Brief pulse treatment, older age, shorter duration of the current depressive episode and psychosis predicted fast remission, but also a lower executive function at baseline as measured with letter 